\title{
Small probability space formulation of Bell's theorem
}

\author{
Márton Gömöri \\ Hungarian Academy of Sciences \\ Budapest, Hungary \\ Tomasz Placek \\ Jagiellonian University \\ Kraków, Poland \\ January 13, 2017
}

To László E. Szabó

\begin{abstract}
A small probability space representation of quantum mechanical probabilities is defined as a collection of Kolmogorovian probability spaces, each of which is associated with a context of a maximal set of compatible measurements, that portrays quantum probabilities as Kolmogorovian probabilities of classical events. Bell's theorem is stated and analyzed in terms of the small probability space formalism.
\end{abstract}




\section{Introduction}

There are two remarkably different ways in which Bell's inequalities can be obtained. Following Bell's original reasoning, the standard approach is to derive Bell's inequalities from the requirement of a common causal explanation for a certain set of events and their probabilities (Bell 1964, 1975; Clauser and Shimony 1978; Hofer-Szabó, Rédei and Szabó 2013, Ch. 9). ${ }^{1}$ The other approach, based on the results of Pitowsky and Fine, does not invoke causal notions but derives Bell's inequalities from the requirement of a certain set of probability-like numbers being representable in a Kolmogorovian probability space (Fine 1982a, 1982b, Accardi 1984; Pitowsky 1989).

Bell's inequalities are famously violated by a certain set of quantum mechanical probabilities that have been measured and confirmed in various experiments. The standard approach and the Pitowsky-Fine approach differ remarkably in what this fact is taken to express. According to the standard approach the violation of Bell's inequalities implies that there is no common causal explanation (of the required sort) for events described by the quantum mechanical probabilities in question. According to the Pitowsky-Fine approach the violation of Bell's inequalities implies that there are no events representable in a Kolmogorovian probability space whose probabilities would be equal to the quantum mechanical probabilities in question.

There is an apparent tension between these two conclusions. If, according to the Pitowsky-Fine approach, there are no events representable in a Kolmogorovian probability space whose probabilities are equal to the quantum mechanical probabilities in question, then what sort of events and what sort of probabilities attached to them are required to be explainable in terms of common causes along the lines of the standard approach? Before answering this question the standard version of Bell's theorem cannot be meaningfully formulated.

This point was first raised by Szabó (1995). His reading of the violation of Bell's inequalities à la Pitowsky-Fine is that there cannot exist events in the

\footnotetext{
${ }^{1}$ Bell's original 1964 derivation proceeds in two steps: first, he refers to the EPR argument as an implication from certain predictions of quantum mechanics to deterministic hidden variables, and second, he shows that deterministic hidden variables imply the Bell inequalities. Despite appearances, as is argued by Gömöri and Hofer-Szabó (2017), the EPR argument is essentially the expression of the need for a common casual explanation for the EPR correlations with EPR's famous Criterion of Reality being a special case of the Common Cause Principle.
} 
world whose probabilities are equal to the quantum mechanical probabilities in question. However, there is a sense in which quantum probabilities reflect the probabilities of real events: Szabó's proposal is that quantum probabilities are conditional in nature. That is, whenever we say $\left\langle\psi, P_{A} \psi\right\rangle$ we do not refer to a probability $p(A)$ of an event $A$, but rather to a conditional probability $p(A \mid a)$, which is to be read as the probability of a measurement outcome $A$ given that the corresponding measurement $a$ has been performed. This understanding of quantum mechanical probabilities is in harmony with the laboratory practice of testing the predictions of quantum mechanics.

In accordance with this interpretation, Szabó proves that there exists a large Kolmogorovian probability space $\Sigma$ in which the quantum mechanical probabilities that violate Bell's inequalities can be represented as conditional probabilities. This space is large insofar as it not only contains events representing outcomes of measurements but also events representing measurement settings. It is in terms of this probability space $\Sigma$ that the requirement of common casual explanation can be meaningfully formulated and the standard version of Bell's theorem can be stated. For, according to Szabó's proposal, the events and probabilities to be explained reside in probability space $\Sigma$.

In the present paper we suggest a slightly different framework for the formulation of Bell's theorem. The philosophy is taken from Szabó: quantum probabilities are not absolute probabilities but probabilities relativized to different measurements. However, instead of starting out from one large probability space $\Sigma$ we shall use a collection of small probability spaces $\left\{\Sigma_{i}\right\}$ each of which is associated with a certain measurement context. ${ }^{2}$ The motivation for this is the following. The large probability space $\Sigma$ includes the measurement settings and their probabilities. These data may vary from experiment to experiment, so we have a different $\Sigma$ space for each instance of the kind of experiment in question. What all these different $\Sigma$ spaces will agree on - and this has been confirmed in experiments of diverse sort-are the conditional probabilities representing the quantum mechanical probabilities. It is thus reasonable to see those conditional probabilities, the robust part of the data, as encoding inherent physical properties of the system in question, as opposed to the probabilities of measurement settings, the varying part of the data, determined by conditions coming from outside of the

\footnotetext{
${ }^{2}$ The distinction large vs. small probability spaces was introduced in the context of Bell's theorem in Butterfield (1992), which also contains the first discussion of the corresponding two kinds of models for quantum correlations.
} 
system, for example by the experimenter's decisions. In this sense the probabilities of measurement settings correspond to an "excess structure" of $\Sigma$ that is not inherent to quantum mechanical phenomena and that we do not expect to be accounted for by our common causal explanation. It is when this sort of excess structure is factored out from Szabó's large probability space that we arrive at the small probability space models of quantum mechanical probabilities. $^{3}$

The paper is structured as follows. In Section 2 we formulate the precise definitions of representations of quantum probabilities in large versus small probability spaces and investigate their relationship. In Section 3 we construct the representation of quantum probabilities associated with the Bell-Aspect experiment in small probability spaces. To our knowledge, this has never been explicitly done in the literature (although the topic of large versus small probability spaces for quantum experiments has been discussed, see Fine 1982a, 1982b; Butterfield 1992; Svetlichny et al. 1988; Müller and Placek 2001). In Section 4 we characterize the requirement of common causal explanation as the existence of a hidden variable model consisting of a further collection of small probability spaces. We will describe these spaces for the Bell-Aspect experiment. Finally, in Section 5 we formulate the standard version of Bell's theorem and connect it with the Pitowsky-Fine version in terms of small probability spaces. More specifically, we demonstrate that the three standard premises of Bell's theorem, Parameter Independence (PI), Outcome Independence (OI), and No Conspiracy (NOCONS), permit one to paste together the small probability spaces representing the Bell-Aspect experiment into one Kolmogorovian probability space, a Kolmogorovian probability representation of this experiment in the original sense of Pitowsky-Fine. Since the latter is not to be had (because of the violation of Bell's inequalities and Pitowsky's theorem), OI, PI, and NOCONS cannot be jointly true.

\section{Representing quantum probabilities}

Definition 1. A quantum mechanical experiment is a triple $\left\langle\mathcal{H}, \psi, \mathcal{O}_{n}\right\rangle$ where $\mathcal{H}$ is a Hilbert space, $\psi \in \mathcal{H}$ is a unit vector, and $\mathcal{O}_{n}=\left\{P_{1}, \ldots, P_{n}\right\}$ is a set

\footnotetext{
${ }^{3}$ Note that this kind of factorization by no means involves assumption about the autonomy of measurement choice in the sense of no-conspiracy. This assumption will be expressed in terms of the small probability space models in Section 4.
} 
of $n$ projection operators on $\mathcal{H}^{4}$

A quantum mechanical experiment represents a finite collection of "yes/no" type quantum mechanical measurements performed in a given quantum state. By "quantum probabilities" we mean the numbers delivered by the quantum mechanical statistical algorithm:

$$
p_{i}=\left\langle\psi, P_{i} \psi\right\rangle
$$

and

$$
p_{i j \ldots k}=\left\langle\psi, P_{i} P_{j} \ldots P_{k} \psi\right\rangle
$$

whenever $P_{i}, P_{j}, \ldots, P_{k}$ pairwise commute. Such a data set of quantum probabilities $\left\langle p_{1}, \ldots, p_{n}, \ldots, p_{i j \ldots k}, \ldots\right\rangle$ will be referred to as the correlation vector associated with the quantum mechanical experiment $\left\langle\mathcal{H}, \psi, \mathcal{O}_{n}\right\rangle$ in question.

A correlation vector associated with a quantum mechanical experiment consists of probability-like numbers. But are they really probabilities? Are there events in the world whose probabilities are equal to quantum probabilities? More precisely, are there events occurring in the runs of a real-world experiment whose relative frequencies are equal to quantum probabilities? A minimal condition for this to be the case is that one has to be able to construct a Kolmogorovian probability space in which those events and their probabilities are accommodated. ${ }^{5}$

Definition 2. A Kolmogorovian representation of a quantum mechanical experiment $\left\langle\mathcal{H}, \psi, \mathcal{O}_{n}\right\rangle$ is a Kolmogorovian probability space $\langle\Omega, \mathcal{F}, p\rangle$ that satisfies the following conditions:

- to each $P_{i} \in \mathcal{O}_{n}$ there corresponds an $\tilde{A}_{i} \in \mathcal{F}$ such that

$$
p\left(\tilde{A}_{i}\right)=\left\langle\psi, P_{i} \psi\right\rangle
$$

- for all $P_{i}, P_{j} \ldots, P_{k} \in \mathcal{O}_{n}$, if they pairwise commute, then

$$
p\left(\tilde{A}_{i} \cap \tilde{A}_{j} \cap \ldots \cap \tilde{A}_{k}\right)=\left\langle\psi, P_{i} P_{j} \ldots P_{k} \psi\right\rangle
$$

\footnotetext{
${ }^{4}$ This definition is taken from Feintzeig 2014.

${ }^{5}$ For more on this point see Szabó's (2001) Laboratory Record Argument.
} 
As we know from Pitowsky (1989), a quantum mechanical experiment whose associated correlation vector violates the appropriate Bell-type inequalities does not have a Kolmogorovian representation. The correlation vector

$$
\left\langle p_{1}, p_{2}, p_{3}, p_{4}, p_{13}, p_{14}, p_{23}, p_{24}\right\rangle=\left\langle\frac{1}{2}, \frac{1}{2}, \frac{1}{2}, \frac{1}{2}, \frac{3}{8}, \frac{3}{8}, 0, \frac{3}{8}\right\rangle
$$

associated with the Bell-Aspect experiment does violate the appropriate Belltype inequalities. Consequently:

Theorem 1. There is a quantum mechanical experiment with no Kolmogorovian representation.

Thus, quantum probabilities are not Kolmogorovian in general. If not Kolmogorovian, then what sort of probabilities are they?

According to Szabó's (1995, 2001) proposal quantum probabilities are Kolmogorovian conditional probabilities.

Definition 3. A large conditional Kolmogorovian representation of a quantum mechanical experiment $\left\langle\mathcal{H}, \psi, \mathcal{O}_{n}\right\rangle$ is a Kolmorogovian probability space $\langle\Omega, \mathcal{F}, p\rangle$ that satisfies the following conditions:

- to each $P_{i} \in \mathcal{O}_{n}$ there corresponds two events $A_{i}, a_{i} \in \mathcal{F}, A_{i} \subset a_{i}$ such that

$$
p\left(A_{i} \mid a_{i}\right)=\left\langle\psi, P_{i} \psi\right\rangle
$$

- for all $P_{i}, P_{j} \in \mathcal{O}_{n}$, if they do not commute, then $a_{i} \cap a_{j}=\emptyset$

- for all $P_{i}, P_{j} \ldots, P_{k} \in \mathcal{O}_{n}$, if they pairwise commute, then $p\left(a_{i} \cap a_{j} \cap \ldots \cap a_{k}\right)>0$ and

$$
p\left(A_{i}^{ \pm} \cap A_{j}^{ \pm} \cap \ldots \cap A_{k}^{ \pm} \mid a_{i} \cap a_{j} \cap \ldots \cap a_{k}\right)=\left\langle\psi, P_{i}^{ \pm} P_{j}^{ \pm} \ldots P_{k}^{ \pm} \psi\right\rangle
$$

where $A_{i}^{+}=A_{i}, A_{i}^{-}=a_{i} \backslash A_{i}, P_{i}^{+}=P_{i}, P_{i}^{-}=P_{i}^{\perp} ; P_{i}^{\perp}$ is the orthogonal complement of $P_{i}$.

Here $a_{i}$ is to be read as the event of setting up a measurement device to perform a certain "yes/no" type measurement, and $A_{i}$ as the event of the firing of the corresponding "yes" detector. The "incompatibility" of two measurements is coded in the postulate that the corresponding two measurement settings are disjoint. 
Since the large probability space $\langle\Omega, \mathcal{F}, p\rangle$ is Kolmogorovian, the probabilities of events in it can be interpreted as relative frequencies measured in a real-world experiment. In contrast, the values of conditional probabilities (4)-(5) that stand for the quantum mechanical probabilities may well turn out not to have a Kolmogorovian representation since they are conditional probabilities with conditioning on different events (as opposed to conditioning on one single event). The values of conditional probabilities pertaining to different conditions typically cannot be delivered (as absolute probabilities) by a single Kolmogorovian probability measure. This explains why correlation vectors like (3), associated with the Bell-Aspect experiment, do not have a Kolmogorovian representation. This is because (3) is in fact a collection of conditional probabilities

$$
\begin{array}{r}
\left\langle p\left(A_{1} \mid a_{1}\right), p\left(A_{2} \mid a_{2}\right), p\left(A_{3} \mid a_{3}\right), p\left(A_{4} \mid a_{4}\right), p\left(A_{1} \cap A_{3} \mid a_{1} \cap a_{3}\right),\right. \\
\left.\quad p\left(A_{1} \cap A_{4} \mid a_{1} \cap a_{4}\right), p\left(A_{2} \cap A_{3} \mid a_{2} \cap a_{3}\right), p\left(A_{2} \cap A_{4} \mid a_{2} \cap a_{4}\right)\right\rangle
\end{array}
$$

that do not belong to the same condition.

Now we formulate an alternative characterization of the sense in which quantum probabilities can be regarded as "conditional".

Definition 4. A small Kolmogorovian representation of a quantum mechanical experiment $\left\langle\mathcal{H}, \psi, \mathcal{O}_{n}\right\rangle$ is a finite collection of Kolmogorovian probability spaces $\left\{\left\langle\Omega_{\alpha}, \mathcal{F}_{\alpha}, p_{\alpha}\right\rangle\right\}_{\alpha \in I}$ that satisfies the following conditions:

- for $\alpha \neq \beta, \Omega_{\alpha} \cap \Omega_{\beta}=\emptyset$

- to each maximal set of pairwise commuting projection operators $\mathcal{O} \subseteq \mathcal{O}_{n}$ there corresponds a Kolmogorovian probability space $\left\langle\Omega_{\alpha}, \mathcal{F}_{\alpha}, p_{\alpha}\right\rangle(\alpha \in I)$ such that $\left\langle\Omega_{\alpha}, \mathcal{F}_{\alpha}, p_{\alpha}\right\rangle$ is a Kolmogorovian representation of the quantum mechanical experiment $\langle\mathcal{H}, \psi, \mathcal{O}\rangle$

Here a probability space $\left\langle\Omega_{\alpha}, \mathcal{F}_{\alpha}, p_{\alpha}\right\rangle$ represents the probabilities of measurement outcomes in a certain measurement context. The contexts are provided by the maximal conjunctions of measurements in the quantum mechanical experiment in question which are (and a fortiori can be) performed simultaneously. In other words, $\left\langle\Omega_{\alpha}, \mathcal{F}_{\alpha}, p_{\alpha}\right\rangle$ encodes the relative frequencies of outcomes in a real-world experiment over the subensemble of experimental runs where a given maximal conjunction of measurements is performed.

Although each small probability space $\left\langle\Omega_{\alpha}, \mathcal{F}_{\alpha}, p_{\alpha}\right\rangle$ alone is Kolmogorovian, quantum probabilities pertaining to different maximal sets 
of compatible measurements reside in different members of the collection $\left\{\left\langle\Omega_{\alpha}, \mathcal{F}_{\alpha}, p_{\alpha}\right\rangle\right\}_{\alpha \in I}$. Probability values belonging to different Kolmogorovian probability spaces do not make up a Kolmogorovian probability measure in general. This is another way to see why correlation vectors like (3) do not have a Kolmogorovian representation. It is because (3) is in fact a collection of probabilities

$$
\begin{aligned}
& \left\langle p_{1 j}\left(A_{1}\right), p_{2 j}\left(A_{2}\right), p_{i 3}\left(A_{3}\right), p_{i 4}\left(A_{4}\right),\right. \\
& \left.p_{13}\left(A_{1} \cap A_{3}\right), p_{14}\left(A_{1} \cap A_{4}\right), p_{23}\left(A_{2} \cap A_{3}\right), p_{24}\left(A_{2} \cap A_{4}\right)\right\rangle
\end{aligned}
$$

that come from different probability spaces - labeled by the pairs of compatible measurements $(i=1,2 ; j=3,4){ }^{6}$

A large conditional Kolmogorovian representation portrays quantum probabilities as conditional probabilities in a large probability space. A small Kolmogorovian representation portrays quantum probabilities as absolute probabilities in many small probability spaces. We shall now demonstrate that large conditional and small Kolmogorovian representations are mutually translatable, though the translation from many small spaces to one large space requires some extra data. First we show that there is a natural translation between large and small Kolmogorovian probability spaces.

Proposition 1. Let $\langle\Omega, \mathcal{F}, p\rangle$ be a Kolmogorovian probability space and $\left\{\Omega_{\alpha}\right\}_{\alpha \in I} \subseteq \mathcal{F}$ such that $p\left(\Omega_{\alpha}\right)>0$. Define $\mathcal{F}_{\alpha}:=\left\{X \subseteq \Omega_{\alpha} \mid X \in \mathcal{F}\right\}$ and $p_{\alpha}(X):=p\left(X \mid \Omega_{\alpha}\right)$ for $X \in \mathcal{F}_{\alpha}$. Then $\left\{\left\langle\Omega_{\alpha}, \mathcal{F}_{\alpha}, p_{\alpha}\right\rangle\right\}_{\alpha \in I}$ is a collection of Kolmogorovian probability spaces.

Proof. It is immediate to see that $\mathcal{F}_{\alpha}$ is a field of subsets of $\Omega_{\alpha}$. As for the satisfaction of Kolmogorov's axioms, clearly $p_{\alpha}\left(\Omega_{\alpha}\right)=1$. For the additivity axiom, let $\mathcal{G}$ be a countable family of pairwise disjoint elements of $\mathcal{F}_{\alpha}$; then $p_{\alpha}(\cup \mathcal{G})=p\left(\cup \mathcal{G} \mid \Omega_{\alpha}\right)=p\left(\cup \mathcal{G} \cap \Omega_{\alpha}\right) / p\left(\Omega_{\alpha}\right)=\sum_{A \in \mathcal{G}} p\left(A \cap \Omega_{\alpha}\right) / p\left(\Omega_{\alpha}\right)=$ $\sum_{A \in \mathcal{G}} p\left(A \mid \Omega_{\alpha}\right)=\sum_{A \in \mathcal{G}} p_{\alpha}(A)$.

Proposition 2. Let $\left\{\left\langle\Omega_{\alpha}, \mathcal{F}_{\alpha}, p_{\alpha}\right\rangle\right\}_{\alpha \in I}$ be a finite collection of Kolmogorovian probability spaces and $\left\{c_{\alpha}\right\}_{\alpha \in I}$ a set of positive coefficients that sum up to 1 . Then $\langle\Omega, \mathcal{F}, p\rangle$ is a Kolmogorovian probability space, where

\footnotetext{
${ }^{6}$ The idea of small Kolmogorovian representation goes back to Kolmogorov (1933) at least, who argued that each experimental arrangement generates its own probability space. Kolmogorov was clear that one should not expect that probabilities of outcomes obtained in various different experimental arrangements can be represented, as absolute probabilities, in a single (perhaps huge) probability space (Khrennikov 2010, p. 26).
} 
- $\Omega=\bigcup_{\alpha \in I} \Omega_{\alpha}$

- for $E \subseteq \Omega, E \in \mathcal{F}$ iff $E \cap \Omega_{\alpha} \in \mathcal{F}_{\alpha}$

- for $E \in \mathcal{F}, p(E)=\sum_{\alpha \in I} c_{\alpha} \cdot p_{\alpha}\left(E \cap \Omega_{\alpha}\right)$

Proof. It is straightforward to see that $\mathcal{F}$ is a field, indeed. To check the axioms, let us calculate first $p(\Omega)=\sum_{\alpha \in I} c_{\alpha} \cdot p_{\alpha}\left(\Omega \cap \Omega_{\alpha}\right)=\sum_{\alpha \in I} c_{\alpha} \cdot p_{\alpha}\left(\Omega_{\alpha}\right)=$ $\sum_{\alpha \in I} c_{\alpha}=1$. Next, let $\mathcal{G}$ be a countable family of pairwise disjoint elements of $\mathcal{F}, p(\cup \mathcal{G})=\sum_{\alpha \in I} c_{\alpha} \cdot p_{\alpha}\left(\cup \mathcal{G} \cap \Omega_{\alpha}\right)=\sum_{\alpha \in I} c_{\alpha} \cdot p_{\alpha}\left(\bigcup_{A \in \mathcal{G}}\left(A \cap \Omega_{\alpha}\right)\right)=$ $\sum_{\alpha \in I} \sum_{A \in \mathcal{G}} c_{\alpha} p_{\alpha}\left(A \cap \Omega_{\alpha}\right)=\sum_{A \in \mathcal{G}} \sum_{\alpha \in I} c_{\alpha} p_{\alpha}\left(A \cap \Omega_{\alpha}\right)=\sum_{A \in \mathcal{G}} p(A)$.

Note that the original (" $p_{\alpha}$ ") probabilities are returned by conditionalization: if $X$ belongs to $\mathcal{F}_{\alpha}$, then $p_{\alpha}(X)=p\left(X \mid \Omega_{\alpha}\right)=p(X) / p\left(\Omega_{\alpha}\right)=$ $c_{\alpha} p_{\alpha}(X) / c_{\alpha}=p_{\alpha}(X){ }^{7}$

This natural translation allows us to construct small Kolmogorovian representations from large ones, and vica versa.

Proposition 3. A quantum mechanical experiment has a large conditional Kolmogorovian representations iff it has a small Kolmogorovian representation.

Proof. (a) Suppose that $\langle\Omega, \mathcal{F}, p\rangle$ is a large conditional Kolmogorovian representation of $\left\langle\mathcal{H}, \psi, \mathcal{O}_{n}\right\rangle$. Consider the collection of Kolmogorovian probability spaces $\left\{\left\langle\Omega_{\alpha}, \mathcal{F}_{\alpha}, p_{\alpha}\right\rangle\right\}_{\alpha \in I}$ constructed from $\langle\Omega, \mathcal{F}, p\rangle$ in Proposition 1 with $\Omega_{\alpha}=a_{m} \cap a_{n} \cap \ldots \cap a_{s}$. Here, following the notation of Definition 3, $a_{m} \cap a_{n} \cap \ldots \cap a_{s}$ corresponds to a maximal conjunction of compatible measurements in $\left\langle\mathcal{H}, \psi, \mathcal{O}_{n}\right\rangle$ as represented in large conditional Kolmogorovian representation $\langle\Omega, \mathcal{F}, p\rangle ; \alpha \in I$ runs over the set of all such conjunctions.

Consider a given $\alpha \in I$. Let $\mathcal{O}_{\alpha}=\left\{P_{m}, P_{n} \ldots, P_{s}\right\} \subseteq \mathcal{O}_{n}$ be the corresponding maximal set of commuting projectors. We demonstrate that $\left\langle\Omega_{\alpha}, \mathcal{F}_{\alpha}, p_{\alpha}\right\rangle$ is a Kolmogorovian representation of $\left\langle\mathcal{H}, \psi, \mathcal{O}_{\alpha}\right\rangle$. Let $\tilde{A}_{i}=$ $A_{i} \cap \Omega_{\alpha}=A_{i} \cap a_{m} \cap a_{n} \cap \ldots \cap a_{s}$ where $A_{i} \in \mathcal{F}$ is the "outcome" set representing $P_{i} \in \mathcal{O}_{\alpha}$ in $\langle\Omega, \mathcal{F}, p\rangle$. Obviously, $\tilde{A}_{i} \in \mathcal{F}_{\alpha}$. The following shows that $\tilde{A}_{i}$ is a representation of $P_{i}$ in $\left\langle\Omega_{\alpha}, \mathcal{F}_{\alpha}, p_{\alpha}\right\rangle$ in the sense of (1):

$$
\begin{aligned}
p_{\alpha}\left(\tilde{A}_{i}\right) & =p\left(\tilde{A}_{i} \mid \Omega_{\alpha}\right)=p\left(A_{i} \cap \Omega_{\alpha} \mid \Omega_{\alpha}\right) \\
& =p\left(A_{i} \mid a_{i} \cap a_{j} \cap \ldots \cap a_{k}\right) \stackrel{\star}{=} p\left(A_{i} \mid a_{i}\right)=\left\langle\psi, P_{i} \psi\right\rangle
\end{aligned}
$$

\footnotetext{
${ }^{7}$ Thanks to Zalán Gyenis for discussions on this topic.
} 
Further, let $P_{i}, P_{j}, \ldots, P_{k} \in \mathcal{O}_{\alpha}$. Then:

$$
\begin{aligned}
p_{\alpha}\left(\tilde{A}_{i} \cap \tilde{A}_{j} \cap \ldots \cap \tilde{A}_{k}\right) & =p\left(\tilde{A}_{i} \cap \tilde{A}_{j} \cap \ldots \cap \tilde{A}_{k} \mid \Omega_{\alpha}\right) \\
& =p\left(A_{i} \cap A_{j} \cap \ldots \cap A_{k} \cap \Omega_{\alpha} \mid \Omega_{\alpha}\right) \\
& =p\left(A_{i} \cap A_{j} \cap \ldots \cap A_{k} \mid a_{m} \cap a_{n} \cap \ldots \cap a_{s}\right) \\
& \stackrel{\star}{=} p\left(A_{i} \cap A_{j} \cap \ldots \cap A_{k} \mid a_{i} \cap a_{j} \cap \ldots \cap a_{k}\right) \\
& =\left\langle\psi, P_{i} P_{j} \ldots P_{k} \psi\right\rangle
\end{aligned}
$$

that is (2) is also satisfied.

In the equalities with $\star$ we made use of the fact that "the statistics of outcomes of a given set of measurements is not altered by performing a further measurement". This is a consequence of the fact that in a commutative sublattice of a Hilbert lattice the law of total probability holds. For example, for all $P_{i}, P_{j} \in \mathcal{O}_{\alpha}$ :

$$
\begin{aligned}
p\left(A_{i} \mid a_{i}\right) & =\left\langle\psi, P_{i} \psi\right\rangle=\left\langle\psi, P_{i} P_{j} \psi\right\rangle+\left\langle\psi, P_{i} P_{j}^{\perp} \psi\right\rangle \\
& =p\left(A_{i} \cap A_{j} \mid a_{i} \cap a_{j}\right)+p\left(A_{i} \cap\left(a_{j} \backslash A_{j}\right) \mid a_{i} \cap a_{j}\right) \\
& =p\left(A_{i} \mid a_{i} \cap a_{j}\right)
\end{aligned}
$$

The derivation is similar for more complex conjunctions of measurements.

(b) Suppose that $\left\{\left\langle\Omega_{\alpha}, \mathcal{F}_{\alpha}, p_{\alpha}\right\rangle\right\}_{\alpha \in I}$ is a small Kolmogorovian representation of $\left\langle\mathcal{H}, \psi, \mathcal{O}_{n}\right\rangle$. Consider the Kolmogorovian probability space $\langle\Omega, \mathcal{F}, p\rangle$ constructed from $\left\{\left\langle\Omega_{\alpha}, \mathcal{F}_{\alpha}, p_{\alpha}\right\rangle\right\}_{\alpha \in I}$ in Proposition 2 with an arbitrary set of positive coefficients $\left\{c_{\alpha}\right\}_{\alpha \in I}$ that sum up to 1 . We demonstrate that $\langle\Omega, \mathcal{F}, p\rangle$ is a large conditional Kolmogorovian representation of $\left\langle\mathcal{H}, \psi, \mathcal{O}_{n}\right\rangle$.

First, consider a $P_{i} \in \mathcal{O}_{n}$. Let

$$
\begin{aligned}
& A_{i}=\bigcup_{\alpha: P_{i} \in \mathcal{O}_{\alpha}} A_{i}^{\alpha} \\
& a_{i}=\bigcup_{\alpha: P_{i} \in \mathcal{O}_{\alpha}} \Omega_{\alpha}
\end{aligned}
$$

where $A_{i}^{\alpha} \in \mathcal{F}_{\alpha}$ is the representing set of $P_{i}$ in $\left\langle\Omega_{\alpha}, \mathcal{F}_{\alpha}, p_{\alpha}\right\rangle$. Obviously, $A_{i}, a_{i} \in \mathcal{F}$. We show that $A_{i}, a_{i}$ provide a "conditional representation" of $P_{i}$ in $\langle\Omega, \mathcal{F}, p\rangle$ in the sense that (4) is satisfied.

$$
p\left(A_{i} \mid a_{i}\right)=p\left(\bigcup_{\alpha: P_{i} \in \mathcal{O}_{\alpha}} A_{i}^{\alpha} \mid \bigcup_{\alpha: P_{i} \in \mathcal{O}_{\alpha}} \Omega_{\alpha}\right)=\frac{p\left(\underset{\alpha: P_{i} \in \mathcal{O}_{\alpha}}{\bigcup_{i}^{\alpha}}\right)}{p\left(\bigcup_{\alpha: P_{i} \in \mathcal{O}_{\alpha}} \Omega_{\alpha}\right)}
$$


As for the numerator,

$$
\begin{aligned}
p\left(\bigcup_{\alpha: P_{i} \in \mathcal{O}_{\alpha}} A_{i}^{\alpha}\right) & =\sum_{\beta \in I} c_{\beta} \cdot p_{\beta}\left(\bigcup_{\alpha: P_{i} \in \mathcal{O}_{\alpha}} A_{i}^{\alpha} \cap \Omega_{\beta}\right) \\
& \stackrel{\star \star}{=} \sum_{\alpha: P_{i} \in \mathcal{O}_{\alpha}} c_{\alpha} \cdot p_{\alpha}\left(A_{i}^{\alpha}\right)=\left(\sum_{\alpha: P_{i} \in \mathcal{O}_{\alpha}} c_{\alpha}\right) \cdot\left\langle\psi, P_{i} \psi\right\rangle
\end{aligned}
$$

as for the denominator,

$$
\begin{aligned}
p\left(\bigcup_{\alpha: P_{i} \in \mathcal{O}_{\alpha}} \Omega_{\alpha}\right) & =\sum_{\beta \in I} c_{\beta} \cdot p_{\beta}\left(\bigcup_{\alpha: P_{i} \in \mathcal{O}_{\alpha}} \Omega_{\alpha} \cap \Omega_{\beta}\right) \\
& \stackrel{\star \star}{=} \sum_{\alpha: P_{i} \in \mathcal{O}_{\alpha}} c_{\alpha} \cdot p_{\alpha}\left(\Omega_{\alpha}\right)=\sum_{\alpha: P_{i} \in \mathcal{O}_{\alpha}} c_{\alpha}
\end{aligned}
$$

Hence, indeed:

$$
p\left(A_{i} \mid a_{i}\right)=\left\langle\psi, P_{i} \psi\right\rangle
$$

In the equalities with $\star \star$ we used that in a small Kolmogorovian representation the $\Omega_{\alpha}$-s are disjoint.

Second, if $P_{i}, P_{j} \in \mathcal{O}_{n}$ do not commute then there is no $\alpha \in I$ such that $P_{i}, P_{j} \in \mathcal{O}_{\alpha}$. Since the $\Omega_{\alpha}$-s are disjoint, then indeed $a_{i} \cap a_{j}=\emptyset$ as required in Definition 3.

Third, suppose that $P_{i}, P_{j} \ldots, P_{k} \in \mathcal{O}_{n}$ pairwise commute. Then there is an $\alpha \in I$ such that $\Omega_{\alpha} \subseteq a_{i} \cap a_{j} \cap \ldots \cap a_{k}$ and hence

$$
p\left(a_{i} \cap a_{j} \cap \ldots \cap a_{k}\right) \geqslant p\left(\Omega_{\alpha}\right)=\sum_{\beta \in I} c_{\beta} \cdot p_{\beta}\left(\Omega_{\alpha} \cap \Omega_{\beta}\right)=c_{\alpha} \cdot p_{\alpha}\left(\Omega_{\alpha}\right)=c_{\alpha}>0
$$

Further, a similar calculation as the single outcome case above yields

$$
p\left(A_{i}^{ \pm} \cap A_{j}^{ \pm} \cap \ldots \cap A_{k}^{ \pm} \mid a_{i} \cap a_{j} \cap \ldots \cap a_{k}\right)=\left\langle\psi, P_{i}^{ \pm} P_{j}^{ \pm} \ldots P_{k}^{ \pm} \psi\right\rangle
$$

That is, (5) is meaningful and satisfied. Note that in this calculation one has to make use of the fact that in a Kolmogorovian representation $\langle\Omega, \mathcal{F}, p\rangle$ not only (2) but also

$$
p\left(\tilde{A}_{i}^{ \pm} \cap \tilde{A}_{j}^{ \pm} \cap \ldots \cap \tilde{A}_{k}^{ \pm}\right)=\left\langle\psi, P_{i}^{ \pm} P_{j}^{ \pm} \ldots P_{k}^{ \pm} \psi\right\rangle
$$

holds, where $\tilde{A}_{i}^{+}=\tilde{A}_{i}, \tilde{A}_{i}^{-}=\Omega \backslash \tilde{A}_{i}$. 
It is a well-known fact of Hilbert space quantum mechanics that a commutative sublattice of a Hilbert lattice is Boolean; further, quantum probability restricted to a Boolean sublattice forms a Kolmogorovian probability measure. This implies that a quantum mechanical experiment $\langle\mathcal{H}, \psi, \mathcal{O}\rangle$ in which all projection operators of $\mathcal{O}$ pairwise commute has a Kolmogorovian representation. This of course remains true when $\mathcal{O}$ is a maximal subset of pairwise commuting projection operators of a set of projectors $\mathcal{O}_{n}$. Therefore, an arbitrary quantum mechanical experiment $\left\langle\mathcal{H}, \psi, \mathcal{O}_{n}\right\rangle$ has a small Kolmogorovian representation. From Proposition 3 it then follows that it also has a large conditional Kolmogorovian representation. Thus:

Theorem 2. All quantum mechanical experiments have large conditional and small Kolmogorovian representations.

That is to say, quantum probabilities, as conditional probabilities in a large space or as absolute probabilities in small spaces, are always Kolmogorovian. This claim can be taken as a version of what Szabó (1995) calls the Kolmogorovian Censorship.

The only difference between large conditional and small Kolmogorovian representations is the extra data of coefficients $\left\{c_{\alpha}\right\}_{\alpha \in I}$ required for building a large space from the collection of small ones. This data determine the probabilities of the measurement settings. In what follows we shall focus on small representations that do not have this excess structure.

The rest of the paper is restricted to the discussion of the Bell-Aspect experiment.

\section{Small Kolmogorovian representation of the Bell-Aspect experiment}

Now we construct a small Kolmogorovian representation of the Bell-Aspect experiment. Consider four non-empty sets $a_{1}, a_{2}, b_{3}, b_{4}$ representing measurement settings on the left (a-settings) and on the right (b-settings), and sub-

sets $A_{i}^{+}, A_{i}^{-} \subseteq a_{i}, B_{j}^{+}, B_{j}^{-} \subseteq b_{j}$ to be thought of as measurement outcomes of $a_{i}$ and $b_{j}$, respectively. We shall use the convention $i \in\{1,2\}$ and $j \in\{3,4\}$, 
and $m, n \in\{-,+\}$. We assume that these sets satisfy the following:

$$
\begin{array}{r}
a_{1} \cap a_{2}=b_{3} \cap b_{4}=\emptyset \\
a_{i} \cap b_{j} \neq \emptyset \\
a_{1} \cup a_{2}=b_{3} \cup b_{4} \\
A_{i}^{-} \cup A_{i}^{+}=a_{i}, \quad B_{j}^{-} \cup B_{j}^{+}=b_{j} \\
A_{i}^{-} \cap A_{i}^{+}=\emptyset, \quad B_{j}^{-} \cap B_{j}^{+}=\emptyset \\
\text { for } i=1,2 ; j=3,4
\end{array}
$$

These set-theoretical requirements express conditions deriving from the physical meaning of the events in question; e.g. that in each run of the experiment a setting on the left and a setting on the right is to be selected, or that $A_{i}^{m}$ cannot occur together with $A_{j}^{m}$ if $i \neq j$. Out of these sets we build now four Kolmogorovian probability spaces, labeled by pairs $\langle i, j\rangle$, in which quantum mechanical probabilities will be represented. We exhibit below the probability space $\left\langle\Omega_{i j}, \mathcal{F}_{i j}, p_{i j}\right\rangle$ :

$$
\begin{array}{r}
\Omega_{i j}=a_{i} \cap b_{j} \\
\mathcal{F}_{i j}=\left\{\emptyset, A_{i}^{+} \cap B_{j}^{+}, A_{i}^{-} \cap B_{j}^{+}, A_{i}^{+} \cap B_{j}^{-}, A_{i}^{-} \cap B_{j}^{-},\right. \\
\left.A_{i}^{+} \cap b_{j}, A_{i}^{-} \cap b_{j}, a_{i} \cap B_{j}^{+}, a_{i} \cap B_{j}^{-}, \Omega_{i j}\right\} \\
p_{i j}\left(A_{i}^{m} \cap b_{j}\right)=\left\langle\psi, P_{i}^{m} \psi\right\rangle p_{i j}\left(a_{i} \cap B_{j}^{n}\right)=\left\langle\psi, P_{j}^{n} \psi\right\rangle \\
p_{i j}\left(A_{i}^{m} \cap B_{j}^{n}\right)=\left\langle\psi, P_{i}^{m} P_{j}^{n} \psi\right\rangle
\end{array}
$$

where $P_{i}^{m}$ and $P_{j}^{n}$ are projections corresponding to outcomes $A_{i}^{m}$ and $B_{j}^{n}$, respectively, and $\psi$ is the singlet state used in the Bell-Aspect experiment. A particular arrangement of the measurement settings yields a probability measure $p_{i j}$ that corresponds to correlation vector (3).

Surface locality, according to which the probability of a measurement outcome in one wing does not depend on the choice of a measurement setting in the other wing, follows from the fact the measures $p_{i j}$ represent quantum probabilities in line with (7). Observe the shape of this condition in the small space formalism:

$$
p_{i j}\left(A_{i}^{m} \cap b_{j}\right)=p_{i j^{\prime}}\left(A_{i}^{m} \cap b_{j^{\prime}}\right) \text { and } p_{i j}\left(a_{i} \cap B_{j}^{n}\right)=p_{i^{\prime} j}\left(a_{i^{\prime}} \cap B_{j}^{n}\right)
$$

The condition relates different probability spaces, as it refers to probability measures belonging to different spaces. In what follows we will come across more constraints relating different small probability spaces. 


\section{Hidden variables: creation of new small probability spaces}

The four Kolmogorovian probability spaces we constructed above offer a probabilistic representation of surface data: of measurement outcomes and their probabilities. In the Bell-Aspect experiment these probabilities are such that there are statistical correlations between the outcomes in the two wings. It is easy to read off from the numbers provided by correlation vector (3) that

$$
p_{i j}\left(\left(A_{i}^{m} \cap b_{j}\right) \cap\left(a_{i} \cap B_{j}^{n}\right)\right) \neq p_{i j}\left(A_{i}^{m} \cap b_{j}\right) p_{i j}\left(a_{i} \cap B_{j}^{n}\right)
$$

Assuming the spatial separation of the two wings, this correlation calls for a common causal explanation. In the hidden variable terminology, a common causal explanation translates to the requirement that the surface data, including the correlations to be explained, emerge from a deeper level of hidden states. We now produce small probability spaces that accommodate these hidden states.

The new probability spaces should deliver probabilities of measurements as marginal probability. In accord with the ideology of the common common cause (Belnap and Szabó 1996), we thus consider a set $\Lambda$, interpreted as the set of all hidden variables, one for all pairs of settings $a_{i}, b_{j}$. With $\Lambda$ we produce four product probability spaces $\left\langle\Omega_{i j \Lambda}, \mathfrak{F}_{i j \Lambda}, \mu_{i j \Lambda}\right\rangle$, where ${ }^{8}$

$$
\Omega_{i j \Lambda}=\left\{A_{i}^{m} \cap B_{j}^{n} \mid m, n \in\{-,+\}\right\} \times \Lambda
$$

$\mathfrak{F}_{i j \Lambda}$ is generated by atoms $\left\{\left\langle A_{i}^{m} \cap B_{j}^{n}, \lambda\right\rangle\right\}$, where $\lambda \in \Lambda$

$$
\mu_{i j \Lambda} \text { satisfies } \sum_{\lambda \in \Lambda} \mu_{i j \lambda}\left(A_{i}^{m} \cap B_{j}^{n}, \lambda\right)=p_{i j}\left(A_{i}^{m} \cap B_{j}^{n}\right)
$$

Observe that the new fields leave no room for some events from the fields of the surface small probability representation, like $a_{i} \cap b_{j}=: \Omega_{i j}$ or $A_{i}^{m} \cap b_{j}$. However, there are in $\mathfrak{F}_{i j \Lambda}$ ersatz objects for such events, as the following "underline" notation explains:

\footnotetext{
${ }^{8}$ To avoid eye strain, below we do not write the angle brackets and curly brackets in parentheses for a probability function.
} 


$$
\begin{gathered}
\left\{\left\langle A_{i}^{m} \cap B_{j}^{n}, \lambda\right\rangle \mid \lambda \in \Lambda\right\}=: \underline{A_{i}^{m} \cap B_{j}^{n}, \Lambda} \\
\left\{\left\langle A_{i}^{m} \cap B_{j}^{n}, \lambda\right\rangle \mid n \in\{-,+\}, \lambda \in \Lambda\right\}=: \underline{A_{i}^{m}} b_{j}, \Lambda \\
\left\{\left\langle A_{i}^{m} \cap B_{j}^{n}, \lambda\right\rangle \mid m \in\{-,+\}, \lambda \in \Lambda\right\}=: \underline{a_{i} B_{j}^{n}, \Lambda} \\
\left\{\left\langle A_{i}^{m} \cap B_{j}^{n}, \lambda\right\rangle \mid m \in\{-,+\}, n \in\{-,+\}, \lambda \in \Lambda\right\}=: \underline{a_{i} b_{j}, \Lambda} \\
\left\{\left\langle A_{i}^{m} \cap B_{j}^{n}, \lambda\right\rangle \mid m \in\{-,+\}, n \in\{-,+\}\right\}=: \underline{a_{i} b_{j}, \lambda}
\end{gathered}
$$

Since we need $\mu$-probabilities conditional on $\lambda$ to be well-defined, we require that $\Lambda$ be countable. Otherwise, we would have $\mu_{i j \Lambda}\left(a_{i} b_{j}, \lambda\right)=0$ for every $\lambda \in \Lambda,{ }^{9}$ making conditional probabilities $\mu_{i j \Lambda}\left(\underline{A_{i}^{m} \cap B_{j}^{n}, \Lambda \mid} \underline{a_{i}} b_{j}, \lambda\right)$ undefined.

The introduction of $\Lambda$ leads to four new product probability spaces, that could be seen as fine-graining of the initial probability spaces $\left\langle\Omega_{i j}, \mathcal{F}_{i j}, p_{i j}\right\rangle$. A large class of such product probability spaces fine-graining a given probability space can be constructed, provided these product spaces are not constrained by further conditions. Causal explanation of the results of the Bell-Aspect experiment imposes, however, such conditions, to which we now turn. We formulate them in the small-space approach.

Outcome Independence (OI) requires that the hidden variable should screen-off the correlation of outcomes registered in separate wings of the experiment. Thus, in the present formulation, it says:

$$
\mu_{i j \Lambda}\left(\underline{A_{i}^{m} \cap B_{j}^{n}, \Lambda}\left|\underline{\left.a_{i} b_{j}, \lambda\right)}=\mu_{i j \Lambda} \underline{\left(A_{i}^{m} b_{j}, \Lambda\right.}\right| \underline{\left.a_{i} b_{j}, \lambda\right)} \times \mu_{i j \Lambda} \underline{\left(a_{i} B_{j}^{n}, \Lambda\right.} \mid \underline{\left.a_{i} b_{j}, \lambda\right)}\right.
$$

Parameter Independence (PI) demands that an outcome in one wing of the experiment should be statistically independent from a setting selected in the other wing given that a hidden state is specified:

$$
\begin{aligned}
& \mu_{i j \Lambda}\left(\underline{A_{i}^{m} b_{j}, \Lambda} \mid \underline{\left.a_{i} b_{j}, \lambda\right)}=\mu_{i j^{\prime} \Lambda}\left(\underline{A_{i}^{m} b_{j^{\prime}}, \Lambda} \mid \underline{a_{i} b_{j^{\prime}}, \lambda}\right)\right. \\
& \mu_{i j \Lambda}\left(\underline{a_{i} B_{j}^{n}, \Lambda} \mid \underline{\left.a_{i} b_{j}, \lambda\right)}=\mu_{i^{\prime} j \Lambda}\left(\underline{a_{i^{\prime}} B_{j}^{n}, \Lambda} \mid \underline{a_{i^{\prime}} b_{j}, \lambda}\right)\right.
\end{aligned}
$$

Finally, No Conspiracy (NOCONS) postulates that pairs of settings be statistically independent from hidden states; in the present formalism, it reads:

$$
\mu_{i j \Lambda}\left(\underline{\left.a_{i} b_{j}, \lambda\right)}=\mu_{i^{\prime} j^{\prime} \Lambda}\left(\underline{a_{i^{\prime}} b_{j^{\prime}}, \lambda}\right)\right.
$$

\footnotetext{
${ }^{9}$ See theorem 10.2 in Billingsley 1995.
} 
(Each condition OI, PI, and NOCONS is universally quantified with respect to $i, i^{\prime} \in\{1,2\}, j, j^{\prime} \in\{3,4\}, m, n \in\{-,+\}$, and $\lambda \in \Lambda$.) Note that in contrast to OI, both PI and NOCONS relate probability measures belonging to different product probability spaces.

To summarize what has been said we state the following definition.

Definition 5. A hidden-state small Kolmogorovian representation of the Bell-Aspect experiment is a quadruple of product probability spaces $\left\langle\Omega_{i j \Lambda}, \mathfrak{F}_{i j \Lambda}, p_{i j \Lambda}\right\rangle(i \in\{1,2\}, j \in\{3,4\})$ of $(8)$, with $\Lambda$ countable and $A_{i}^{m}, B_{j}^{n}, a_{i}, b_{j}$ satisfying (6), and $p_{i j}$ given by $(7)$.

A small-space local common causal non-conspiratorial model of the Bell-Aspect experiment is a hidden-state small Kolmogorovian representation of the Bell-Aspect experiment that satisfies the three conditions OI, PI, and NOCONS.

It is no news that the combination of OI, PI, and NOCONS permits a derivation of Bell's inequalities. It will be instructive to see, however, how this derivation goes through in a prudent framework of small product probability spaces. This will lead us to ask what mathematical meaning the combination of the three conditions has, and the answer we give will take us back to Kolmogorovian representability in the original sense of Pitowsky-Fine described in Definition 2.

\section{The mathematical meaning of OI, PI, and NOCONS}

To examine the mathematical meaning of OI, PI, and NOCONS, it might help to sketch a particular attitude to Bell's theorem. The essence of this attitude is a belief that working with small probability spaces, that is, by first producing a surface small Kolmogorovian representation of the Bell-Aspect experiment, and then fine-graining it to obtain a hidden-state small Kolmogorovian representation, one somehow blocks the derivation of Bell's inequalities. To some extent, this belief is justified: there is a large class of hidden-state small Kolmogorovian representations of the experiment which do not imply Bell's inequalities. But then, dramatically, once OI, PI, and NOCONS are brought into play, the Bell's inequalities become derivable. So, what, mathematically speaking, do the three conditions do to a hidden-state 
small Kolmogorovian representation? The theorem below offers an answer to this query.

Theorem 3. Let $\left\langle\Omega_{i j \Lambda}, \mathfrak{F}_{i j \Lambda}, p_{i j \Lambda}\right\rangle(i \in\{1,2\}, j \in\{3,4\})$ be a hidden-state Kolmogorovian representation of the Bell-Aspect experiment, delivering surface probabilities $p_{i j}$. Assume that the spaces $\left\langle\Omega_{i j \Lambda}, \mathfrak{F}_{i j \Lambda}, p_{i j \Lambda}\right\rangle$ satisfy OI, PI, and NOCONS (i.e., they form a small-space model of Definition 5). Then there is a Kolmogorovian probability space $\langle\Omega, \mathfrak{F}, P\rangle$ such that all the surface probabilities $p_{i j}$ are identifiable with absolute probabilities in $\langle\Omega, \mathfrak{F}, P\rangle .{ }^{10}$

Proof. Let $\left\langle\Omega_{i j \Lambda}, \mathfrak{F}_{i j \Lambda}, p_{i j \Lambda}\right\rangle(i \in\{1,2\}, j \in\{3,4\})$ be as stated in the premise of the theorem. Using an idea of Fine's theorem, by applying the three conditions OI, PI, and NOCONS, we will produce a Kolmogorovian product probability space $\langle\Omega, \mathcal{F}, P\rangle$ that represents probabilities $p_{i j}$ of the small probability spaces as absolute probabilities. The essential part of the argument is that the new probability space returns probabilities of joint and single outcomes, which is a crux of Fine's theorem.

The base set of our product space is:

$$
\Omega=\left\{A_{1}^{-}, A_{1}^{+}\right\} \times\left\{A_{2}^{-}, A_{2}^{+}\right\} \times\left\{B_{3}^{-}, B_{3}^{+}\right\} \times\left\{B_{4}^{-}, B_{4}^{+}\right\}
$$

its field of subsets $\mathcal{F}$ is generated by the following atoms:

$$
\left\{\left\langle A_{1}^{m}, A_{2}^{n}, B_{3}^{r}, B_{4}^{t}\right\rangle\right\} \text {, with } m, n, r, t \in\{+,-\}
$$

and the measure $P$ is given by:

$$
\begin{aligned}
& P\left(\left\langle A_{1}^{m}, A_{2}^{n}, B_{3}^{r}, B_{4}^{t}\right\rangle\right)= \\
& \left.\left.\sum_{\lambda \in \Lambda} \mu_{13 \Lambda} \underline{\left(a_{1} b_{3}, \lambda\right.}\right) \times \mu_{13 \Lambda}\left(\underline{A_{1}^{m} b_{3}, \Lambda} \mid \underline{a_{1} b_{3}, \lambda}\right) \times \mu_{13 \Lambda} \underline{\left(a_{1} B_{3}^{r}, \Lambda\right.} \mid \underline{a_{1} b_{3}, \lambda}\right) \times \\
& \quad \times \mu_{24 \Lambda} \underline{\left.\left(\underline{A_{2}^{n} b_{4}, \Lambda} \mid \underline{a_{2} b_{4}, \lambda}\right) \times \mu_{24 \Lambda} \underline{\left(\underline{a_{2} B_{4}^{t}, \Lambda}\right.} \mid \underline{a_{2} b_{4}, \lambda}\right)}
\end{aligned}
$$

We need to show the satisfaction of equations like

$$
P\left(\left\{\left\langle A_{1}^{m}, A_{2}^{n}, B_{3}^{r}, B_{4}^{t}\right\rangle \mid m, t \in\{-,+\}\right\}\right)=p_{23}\left(A_{2}^{n} \cap B_{3}^{r}\right)
$$

We calculate only this case, observing how OI, PI, and NOCONS permit one to change labels of small probability spaces. In the calculation we indicate

\footnotetext{
${ }^{10} \mathrm{An}$ analogous construction, but in a specific and little known framework of stochastic outcomes in branching spacetimes (SOBST) is carried out in Müller and Placek 2001.
} 
where these conditions are used (we leave other cases as an exercise for the reader).

$$
\begin{aligned}
& P\left(\left\{\left\langle A_{1}^{m}, A_{2}^{n}, B_{3}^{r}, B_{4}^{t}\right\rangle \mid m, t \in\{-,+\}\right\}\right)= \\
& \left.\sum_{\lambda \in \Lambda} \mu_{13 \Lambda}\left(\underline{a_{1} b_{3}, \lambda}\right) \times \mu_{13 \Lambda}\left(\underline{a_{1} b_{3}, \Lambda} \mid \underline{a_{1} b_{3}, \lambda}\right) \times \mu_{13 \Lambda} \underline{\left(a_{1} B_{3}^{r}, \Lambda\right.} \mid \underline{a_{1} b_{3}, \lambda}\right) \times \\
& \times \mu_{24 \Lambda}\left(\underline{A_{2}^{n} b_{4}, \Lambda} \mid \underline{a_{2} b_{4}, \lambda}\right) \times \mu_{24 \Lambda}\left(\underline{a_{2} b_{4}, \Lambda} \mid \underline{a_{2} b_{4}, \lambda}\right)= \\
& \left.\sum_{\lambda \in \Lambda} \mu_{13 \Lambda} \underline{\left(a_{1} b_{3}, \lambda\right)} \times 1 \times \mu_{13 \Lambda}\left(\underline{a_{1} B_{3}^{r}, \Lambda} \mid \underline{a_{1} b_{3}, \lambda}\right) \times \mu_{24 \Lambda} \underline{\left(A_{2}^{n} b_{4}, \Lambda\right.} \mid \underline{a_{2} b_{4}, \lambda}\right) \times 1 \stackrel{\text { NOCONS }}{=} \\
& \left.\left.\sum_{\lambda \in \Lambda} \mu_{23 \Lambda}\left(\underline{a_{2} b_{3}, \lambda}\right) \times \mu_{13 \Lambda} \underline{\left(a_{1} B_{3}^{r}, \Lambda\right.} \mid \underline{a_{1} b_{3}, \lambda}\right) \times \mu_{24 \Lambda} \underline{\left(A_{2}^{n} b_{4}, \Lambda\right.} \mid \underline{a_{2} b_{4}, \lambda}\right) \stackrel{2 \times P I}{=} \\
& \left.\sum_{\lambda \in \Lambda} \mu_{23 \Lambda}\left(\underline{a_{2} b_{3}, \lambda}\right) \times \mu_{23 \Lambda} \underline{\left(\underline{a_{1} B_{3}^{r}, \Lambda}\right.} \mid \underline{a_{2} b_{3}, \lambda}\right) \times \mu_{23 \Lambda}\left(\underline{A_{2}^{n} b_{3}, \Lambda} \mid \underline{a_{2} b_{3}}, \lambda\right) \stackrel{\text { OI }}{=} \\
& \left.\sum_{\lambda \in \Lambda} \mu_{23 \Lambda}\left(\underline{a_{2} b_{3}, \lambda}\right) \times \mu_{23 \Lambda} \underline{\left(A_{2}^{n} \cap B_{3}^{r}, \Lambda\right.} \mid \underline{a_{2} b_{3}, \lambda}\right)= \\
& \mu_{23 \Lambda}\left(\underline{A_{2}^{n} \cap B_{3}^{r}, \Lambda}\right)=p_{23}\left(A_{2}^{n} \cap B_{3}^{r}\right)=\left\langle\psi \mid P_{2}^{n} P_{3}^{r} \psi\right\rangle
\end{aligned}
$$

Finally, note that

$$
\begin{aligned}
& P\left(\left\{\left\langle A_{1}^{m}, A_{2}^{n}, B_{3}^{+}, B_{4}^{t}\right\rangle \mid m, t \in\{-,+\}\right\}\right) \\
& \quad+P\left(\left\{\left\langle A_{1}^{m}, A_{2}^{n}, B_{3}^{-}, B_{4}^{t}\right\rangle \mid m, t \in\{-,+\}\right\}\right)= \\
& P\left(\left\{\left\langle A_{1}^{m}, A_{2}^{n}, B_{3}^{r}, B_{4}^{t}\right\rangle \mid m, r, t \in\{-,+\}\right\}\right)=p_{23}\left(A_{2}^{n} \cap b_{3}\right)=\left\langle\psi \mid P_{2}^{n} \psi\right\rangle
\end{aligned}
$$

and analogously,

$$
P\left(\left\{\left\langle A_{1}^{m}, A_{2}^{n}, B_{3}^{r}, B_{4}^{t}\right\rangle \mid m, n, t \in\{-,+\}\right\}\right)=p_{23}\left(a_{2} \cap B_{3}^{r}\right)=\left\langle\psi \mid P_{3}^{r} \psi\right\rangle
$$

Since $P$ is defined on atoms of $\mathcal{F}$, it is additive by the definition. Equations (15)-(17) together with the definition of probability functions $p_{i j}$ (Eq. (7)) show that $P$ is normalized to unity, i.e., $P\left(\left\{\left\langle A_{1}^{m}, A_{2}^{n}, B_{3}^{r}, B_{4}^{t}\right\rangle \mid\right.\right.$ $m, n, r, t \in\{-,+\}\})=1$. Thus, $P$ is a probability measure, and $\langle\Omega, \mathcal{F}, P\rangle$ is a Kolmogorovian probability space, such that all the surface probabilities $p_{i j}$ are identifiable with absolute probabilities in $\langle\Omega, \mathcal{F}, P\rangle$.

The theorem thus shows that the three conditions OI, PI, NOCONS transform a hidden-state small Kolmogorovian representation of the Bell-Aspect 
experiment into a Kolmogorovian representation of this experiment (of Definition 2): the set $\left\{\left\langle A_{1}^{m}, A_{2}^{n}, B_{3}^{r}, B_{4}^{t}\right\rangle \mid n, r, t \in\{-,+\}\right\}$ corresponds to a single projector $P_{1}^{m}$ present in the experiment, the set $\left\{\left\langle A_{1}^{m}, A_{2}^{n}, B_{3}^{r}, B_{4}^{t}\right\rangle \mid\right.$ $n, t \in\{-,+\}\}$ corresponds to a "double" projector $P_{1}^{m} P_{3}^{r}$ (and analogously for other projectors involved). Thus, whereas from a metaphysical perspective, the three conditions embody a claim about a causal underpinning of the Bell-Aspect experiment, their mathematical meaning is that they enforce pasting together of the four probability spaces (of the hidden-state small Kolmogorovian representation) into one probability space, constituting a Kolmogorovian representation of the Bell-Aspect experiment. By Pitowsky's theorem, the existence of the latter representation implies Bell's inequalities; and, to repeat, these inequalities are violated by quantum mechanics and most likely by Nature as well.

Given the mathematical meaning of the three conditions, one might want to draw a moral which perhaps suggests a further research program (this is a view of one co-author - TP, not shared by the other). Having seen what the three conditions do to a hidden-variable small Kolmogorovian representation, one should modify them so that the modified version do not enforce the pasting together of the small probability spaces. To please our causal intuitions, the modified OI, PI, and NOCONS should bear some relations to ideas underlying Reichenbach's common cause principle, locality, and no conspiracy. Hofer-Szabó's (2008) model with separate screener systems might be perhaps seen as a case in point (for an examination of the model, and some reservations towards it, see Wroński, Placek, and Godziszewski's paper in this volume). The mathematical meaning of the three conditions suggests also a modest program of testing models for the Bell-Aspect experiment, by asking whether the conditions of a given model imply the existence of Kolmogorovian representation of this experiment, or not.

\section{Conclusions}

According to the Pitowsky-Fine approach the violation of Bell's inequalities is the expression of the fact that quantum probabilities are not Kolmogorovian probabilities. However, as we learn from Szabó (1995, 2001), they should not be absolute Kolmogorovian probabilities in the first place: by virtue of their meaning, quantum probabilities are conditional and they reside in a large Kolmogorovian probability space that describes the mea- 
surement events of a real-world experiment.

There is a sense however in which Szabó's large space is not appropriate. Its construction essentially relies on an "excess structure" that does not seem to be inherent to the quantum phenomena. This excess structure corresponds to the probabilities of the different measurement settings that depends on the experimenter's decisions. We examined a simple method that factors out this excess structure to arrive at a collection of small Kolmogorovian probability spaces in which quantum probabilities are represented as absolute probabilities.

The idea that correlations between events residing in these small probability spaces call for a common causal explanation motivates a search for a hidden variable model of the experiment. We characterized such hidden variable models in terms of the small probability space representation of quantum probabilities. We showed that the existence of such a model allows us to paste together the small probability spaces representing the quantum probabilities in question into one single Kolmogorovian probability space that constitutes a Kolmogorovian representation of those quantum probabilities (in the original sense of Pitowsky-Fine).

In conclusion, one can phrase the duality of the two approaches to Bell's theorem this way: while the physical meaning of the violation of Bell's inequalities is the impossibility of certain causal explanations of the quantum phenomenon in question (respecting some physical/metaphysical principles about causation embodied by OI, PI, and NOCONS), the mathematical meaning of these principles is that they produce a Kolmogorovian probability representation of the phenomenon, which cannot exist (by Pitowski's theorem and the violation of Bell's inequalities).

Acknowledgements. This work has been supported by the Hungarian Scientific Research Fund, OTKA K-115593 and by the Bilateral Mobility Grant of the Hungarian and Polish Academies of Sciences, NM-104/2014.

\section{References}

Accardi, L. 1984. The probabilistic roots of the quantum mechanical paradoxes. In The Wave-Particle Dualism, ed. S. Diner et al. Dordrecht: Reidel. 
Bell, J.S. 1964. On the Einsten-Podolsky-Rosen paradox. Physics 1:195. Reprinted in J. S. Bell, Speakable and unspeakable in quantum mechanics. Cambridge: Cambridge University Press, 2004.

Bell, J.S. 1976. The theory of local beables. Epistemological Letters, March 1976. Reprinted in J. S. Bell, Speakable and unspeakable in quantum mechanics. Cambridge: Cambridge University Press, 2004.

Belnap, N. and Szabó, L.E. 1996. Branching Space time analysis of the GHZ theorem. Foundations of Physics 26:989.

Billingsley, P. 1995. Probability and Measure. 3rd edn. New York: John Wiley \& Sons, Inc.

Butterfield, J. 1992. Bell's Theorem: What it Takes. British Journal for the Philosophy of Science 43:41-83.

Clauser, J.F. and Shimony, A. 1978. Bell's theorem: Experimental tests and implications. Reports on Progress in Physics 41:1881.

Feintzeig, B. 2015. Hidden Variables and Incompatible Observables in Quantum Mechanics. The British Journal for the Philosophy of Science 66(4):905-927.

Fine, A. .1982a. Joint Distributions, Quantum Correlations, and Commuting Observables. Journal of Mathematical Physics 23:1306.

Fine, A. 1982b. Hidden Variables, Joint Probability, and the Bell Inequalities. Physical Review Letters 48:291.

Gömöri, M. and Hofer-Szabó, G. 2017. On the meaning of EPR's Criterion of Reality. (In preparation).

Hofer-Szabó, G. 2008. Separate- versus common-common-cause-type derivations of the Bell inequalities. Synthese 163(2):199-215.

Hofer-Szabó, G., Rédei, M. and Szabó, L.E. 2013. The Principle of the Common Cause. Cambridge: Cambridge University Press.

Khrennikov, A.Y. 2010. Ubiquitous Quantum Structure: From psychology to finance. Berlin: Springer. 
Kolmogorov, A.N. 1933. Grundbegriffe der Wahrscheinlichkeitsrechnung. Berlin: Julius Springer. English translation: Kolmogorov, A.N., Foundations of the Theory of Probability. 2nd edn. New York: Chelsea, 1956.

Müller, T. and Placek, T. 2001. Against a Minimalist Reading of Bell's Theorem: Lessons from Fine. Synthese 128(3):343.

Svetlichny, G., Redhead, M., Brown, H. and Butterfield, J. 1988. Do the Bell Inequalities Require the Existence of Joint Probability Distributions? Philosophy of Science 55(3):387.

Szabó, L.E. 1995. Is quantum mechanics compatible with a deterministic universe? Two interpretations of o quantum probabilities. Foundations of Physics Letters 8:417.

Szabó, L.E. 2001. Critical reflections on quantum probability theory. In John von Neumann and the Foundations of Quantum Physics, ed. M. Rédei and M. Stoeltzner, Dordrecht: Kluwer.

Pitowsky, I. 1989. Quantum Probability - Quantum Logic. Berlin: Springer. 